\title{
The Cyclotomic Numbers of Order Sixteen
}

\author{
By Ronald J. Evans and Jay Roderick Hill
}

\begin{abstract}
A complete table of $\mathbf{4 0 8}$ formulas for cyclotomic numbers of order sixteen is presented. Each number is expressed as a linear combination of parameters of quartic, octic, and bioctic Jacobi sums. Recent applications of these formulas are discussed.
\end{abstract}

1. Introduction and Notation. Let $p=16 f+1$ be a prime with a fixed primitive root $g$. Define the cyclotomic number $(i, j)$ of order sixteen to be the number of integers $n(\bmod p)$ which satisfy

$$
n \equiv g^{16 s+i}, \quad 1+n \equiv g^{16 t+j} \quad(\bmod p)
$$

for some choices of $s, t$ in $\{0,1,2, \ldots, f-1\}$. E. Lehmer [10] began the study of these numbers in 1954. A few years later A. L. Whiteman found formulas for the cyclotomic numbers of order sixteen, in terms of parameters of quartic, octic and bioctic Jacobi sums. He gave a table of formulas for $(i, 0)$ in [17]. (The cyclotomic numbers $(i, 0)$ are particularly important, as they have been applied to prove the nonexistence of sixteenth power residue difference sets and modified difference sets; see [17, Section 4], [6].) Most of the formulas for the remaining cyclotomic numbers of order sixteen were not published, and they have apparently been inaccessible. In contrast, complete tables for the cyclotomic numbers of order $k$ are available for $k=$ $2,3,4,5,6[5] ; k=7[14] ; k=8[11] ; k=9,18$ [2]; $k=10,12$ [18], [19]; $k=11[13] ; k=14[15] ; k=20[16]$.

Our objective is to present in Section 3 a complete table of the formulas for the cyclotomic numbers of order sixteen. The computations were performed on the Burroughs 6700 at UCSD, with use of algorithms described in [17, p. 408]. Selected formulas from the tables in Section 3 have been utilized [7] to give elementary resolutions of sign ambiguities in quartic and octic Jacobi and Jacobsthal sums, in certain cases (see Section 2). It is not possible to accomplish these sign resolutions with use of formulas from Whiteman's tables alone. This is what motivated the present work.

We will need the following additional notation. Let $\beta=\exp (2 \pi i / 16)$, and fix a character $\chi(\bmod p)$ of order 16 such that $\chi(g)=\beta$. Let $m$ denote the least positive index of 2 with respect to the base $g$. For any characters $\lambda, \psi(\bmod p)$, define

Received January 7, 1978.

AMS (MOS) subject classifications (1970). Primary 10G05, 10A40, 10G04; Secondary $05 \mathrm{~B} 10$.

Key words and phrases. Cyclotomic numbers of order sixteen, Jacobi sums, Jacobsthal sums, sign ambiguities. 
the Jacobi sum

$$
J(\lambda, \psi)=\sum_{n(\bmod p)} \lambda(n) \psi(1-n) .
$$

In the notation of $[17$, Section 3$]$, we have

$$
\begin{aligned}
J\left(\chi^{4}, \chi^{4}\right) & =-x+2 i y \quad\left(p=x^{2}+4 y^{2}, x \equiv 1(\bmod 4)\right), \\
J\left(\chi^{2}, \chi^{6}\right) & =-a+i b \sqrt{2} \quad\left(p=a^{2}+2 b^{2}, a \equiv 1(\bmod 4)\right), \\
(-1)^{f} J\left(\chi, \chi^{7}\right) & =c_{0}+c_{2} \sqrt{2}+i c_{1} \sqrt{2-\sqrt{2}}+i c_{3} \sqrt{2+\sqrt{2}} \\
& =c_{0}+c_{2}\left(\beta^{2}-\beta^{6}\right)+c_{1}\left(\beta+\beta^{7}\right)+c_{3}\left(\beta^{3}+\beta^{5}\right),
\end{aligned}
$$

and

$$
J\left(\chi, \chi^{2}\right)=\sum_{i=0}^{7} d_{i} \beta^{i}
$$

In Section 3, the numbers $256(i, j)$ are expressed as linear combinations of $p, 1, x$, $y, a, b$, and the $d_{i}$ and $c_{i}$.

TABLE 1a. $f$ even, $m \equiv 0(\bmod 8)$

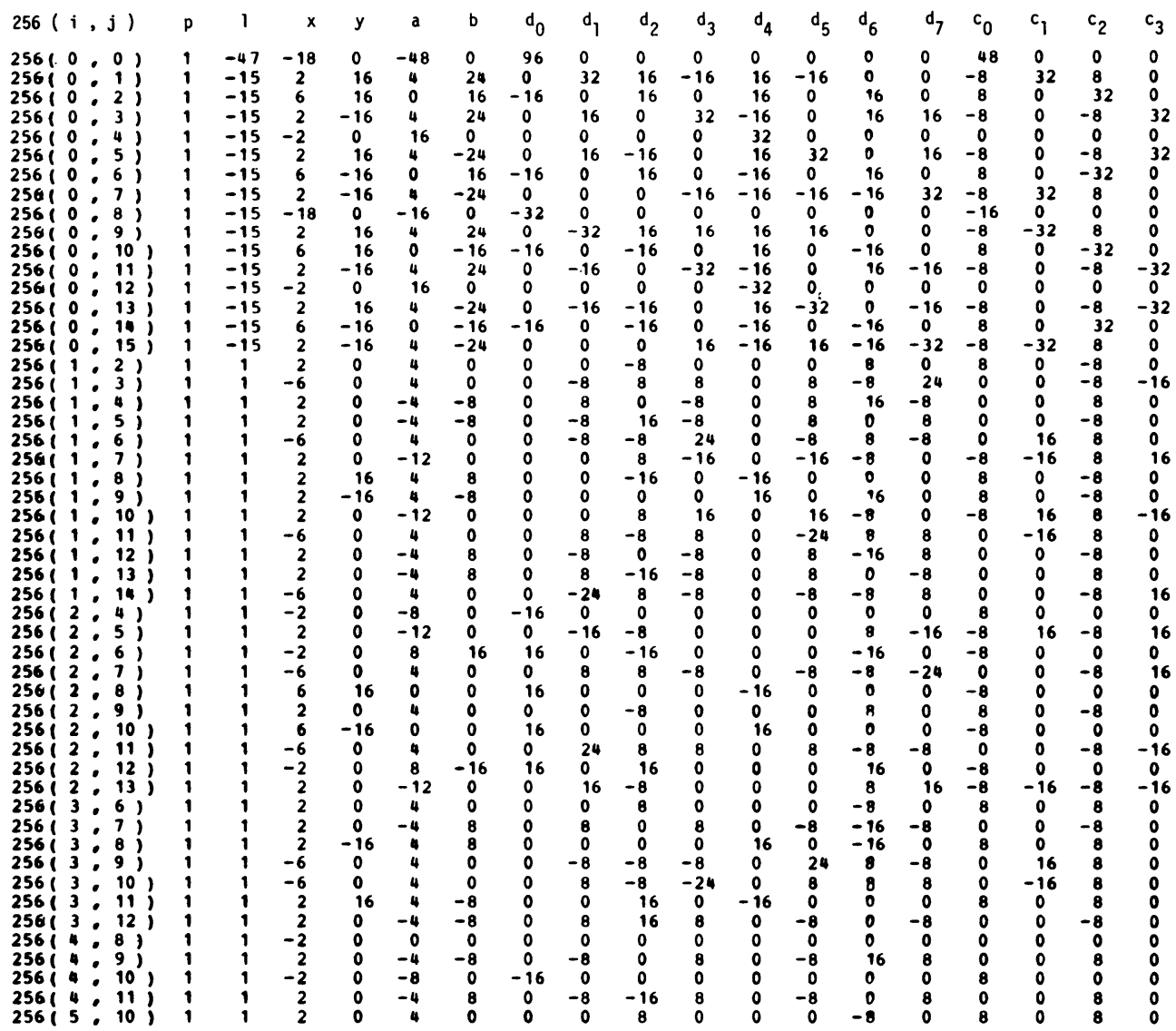


TABLE $1 b . f$ odd $m \equiv 0(\bmod 8)$

\begin{tabular}{|c|c|c|c|c|c|c|c|c|c|c|c|c|c|c|c|c|c|c|c|}
\hline 561 & ,j) & & 1 & $x$ & v & & b & $\mathrm{d}_{0}$ & $d_{1}$ & $d_{2}$ & $d_{3}$ & $d_{4}$ & $d_{5}$ & $d_{6}$ & $d_{7}$ & $\mathrm{c}_{0}$ & $c_{1}$ & $c_{2}$ & $c_{3}$ \\
\hline 2 & 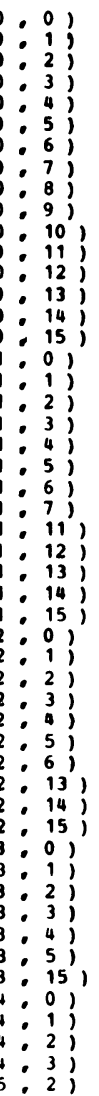 & $\begin{array}{l}1 \\
1 \\
1 \\
1 \\
1 \\
1 \\
1 \\
1 \\
1 \\
1 \\
1 \\
1 \\
1 \\
1 \\
1 \\
1 \\
1 \\
1 \\
1 \\
1 \\
1 \\
1 \\
1 \\
1 \\
1 \\
1 \\
1 \\
1 \\
1 \\
1 \\
1 \\
1 \\
1 \\
1 \\
1 \\
1 \\
1 \\
1 \\
1 \\
1 \\
1 \\
1 \\
1 \\
1 \\
1 \\
1 \\
1 \\
1 \\
1 \\
1 \\
1\end{array}$ & $\begin{array}{c}-31 \\
1 \\
1 \\
1 \\
1 \\
1 \\
1 \\
1 \\
1 \\
1 \\
1 \\
1 \\
1 \\
1 \\
1 \\
-15 \\
-15 \\
1 \\
1 \\
1 \\
1 \\
1 \\
1 \\
1 \\
1 \\
1 \\
1 \\
-15 \\
1 \\
-15 \\
1 \\
1 \\
1 \\
1 \\
1 \\
1 \\
1 \\
-15 \\
1 \\
-15 \\
1 \\
1 \\
1 \\
-15 \\
1 \\
1 \\
1 \\
1\end{array}$ & $\begin{array}{r}-18 \\
2 \\
6 \\
2 \\
-2 \\
2 \\
6 \\
2 \\
-18 \\
2 \\
6 \\
2 \\
-2 \\
2 \\
6 \\
2 \\
2 \\
2 \\
2 \\
-6 \\
2 \\
2 \\
-6 \\
2 \\
-6 \\
2 \\
2 \\
-6 \\
2 \\
6 \\
2 \\
6 \\
-6 \\
-2 \\
2 \\
-2 \\
2 \\
-2 \\
-6 \\
2 \\
-6 \\
-6 \\
2 \\
2 \\
2 \\
2 \\
-2 \\
2 \\
-2 \\
2 \\
2\end{array}$ & $\begin{array}{c}0 \\
16 \\
16 \\
-16 \\
0 \\
16 \\
-16 \\
-16 \\
0 \\
16 \\
16 \\
-16 \\
0 \\
16 \\
-16 \\
-16 \\
16 \\
-16 \\
0 \\
0 \\
0 \\
0 \\
0 \\
0 \\
0 \\
0 \\
0 \\
0 \\
0 \\
16 \\
0 \\
-16 \\
0 \\
0 \\
0 \\
0 \\
0 \\
0 \\
0 \\
-16 \\
0 \\
0 \\
16 \\
0 \\
0 \\
0 \\
0 \\
0 \\
0 \\
0 \\
0\end{array}$ & $\begin{array}{c}-16 \\
4 \\
0 \\
4 \\
16 \\
4 \\
0 \\
4 \\
-48 \\
4 \\
0 \\
4 \\
16 \\
4 \\
0 \\
4 \\
4 \\
4 \\
-12 \\
4 \\
-4 \\
-4 \\
4 \\
4 \\
4 \\
-4 \\
-4 \\
4 \\
-12 \\
0 \\
4 \\
0 \\
4 \\
8 \\
-12 \\
-8 \\
-12 \\
8 \\
4 \\
4 \\
4 \\
4 \\
-4 \\
4 \\
-4 \\
0 \\
-4 \\
-8 \\
-4 \\
4\end{array}$ & $\begin{array}{c}0 \\
24 \\
-16 \\
24 \\
0 \\
-24 \\
-16 \\
-24 \\
0 \\
24 \\
16 \\
24 \\
0 \\
-24 \\
16 \\
-24 \\
8 \\
-8 \\
0 \\
0 \\
8 \\
8 \\
0 \\
0 \\
0 \\
-8 \\
-8 \\
0 \\
0 \\
0 \\
0 \\
0 \\
0 \\
-16 \\
0 \\
0 \\
0 \\
16 \\
0 \\
8 \\
0 \\
0 \\
-8 \\
-8 \\
0 \\
8 \\
0 \\
-8 \\
0 \\
8 \\
0\end{array}$ & $\begin{array}{c}0 \\
0 \\
-16 \\
0 \\
32 \\
0 \\
-16 \\
0 \\
0 \\
0 \\
-16 \\
0 \\
32 \\
0 \\
-16 \\
0 \\
0 \\
0 \\
0 \\
0 \\
0 \\
0 \\
0 \\
0 \\
0 \\
0 \\
0 \\
0 \\
0 \\
16 \\
0 \\
16 \\
0 \\
-16 \\
0 \\
16 \\
0 \\
-16 \\
0 \\
0 \\
0 \\
0 \\
0 \\
0 \\
0 \\
0 \\
-32 \\
0 \\
16 \\
0 \\
0\end{array}$ & $\begin{array}{c}0 \\
0 \\
0 \\
-16 \\
0 \\
16 \\
0 \\
0 \\
0 \\
0 \\
0 \\
16 \\
0 \\
-16 \\
0 \\
0 \\
0 \\
0 \\
-16 \\
-8 \\
24 \\
8 \\
-8 \\
-16 \\
8 \\
8 \\
-8 \\
8 \\
16 \\
0 \\
16 \\
0 \\
8 \\
0 \\
0 \\
0 \\
0 \\
0 \\
-8 \\
0 \\
8 \\
-8 \\
0 \\
8 \\
-16 \\
-24 \\
0 \\
-8 \\
0 \\
-8 \\
16\end{array}$ & $\begin{array}{c}0 \\
-16 \\
-16 \\
0 \\
0 \\
16 \\
-16 \\
0 \\
0 \\
-16 \\
16 \\
0 \\
0 \\
16 \\
16 \\
0 \\
16 \\
0 \\
8 \\
-8 \\
0 \\
16 \\
8 \\
-8 \\
8 \\
0 \\
-16 \\
-8 \\
8 \\
0 \\
-8 \\
0 \\
8 \\
16 \\
-8 \\
0 \\
-8 \\
-16 \\
8 \\
0 \\
-8 \\
-8 \\
-16 \\
-16 \\
8 \\
0 \\
0 \\
0 \\
0 \\
16 \\
8\end{array}$ & $\begin{array}{c}0 \\
16 \\
0 \\
0 \\
0 \\
0 \\
0 \\
-16 \\
0 \\
-16 \\
0 \\
0 \\
0 \\
0 \\
0 \\
16 \\
0 \\
0 \\
0 \\
-8 \\
-8 \\
24 \\
8 \\
16 \\
-8 \\
-8 \\
-8 \\
8 \\
0 \\
0 \\
-16 \\
0 \\
-8 \\
0 \\
16 \\
0 \\
-16 \\
0 \\
8 \\
0 \\
8 \\
-8 \\
0 \\
8 \\
-16 \\
8 \\
0 \\
8 \\
0 \\
-24 \\
16\end{array}$ & $\begin{array}{c}0 \\
16 \\
-16 \\
-16 \\
32 \\
16 \\
16 \\
-16 \\
0 \\
16 \\
-16 \\
-16 \\
-32 \\
16 \\
16 \\
-16 \\
-16 \\
16 \\
0 \\
0 \\
0 \\
0 \\
0 \\
0 \\
0 \\
0 \\
0 \\
0 \\
0 \\
16 \\
0 \\
-16 \\
0 \\
0 \\
0 \\
0 \\
0 \\
0 \\
0 \\
16 \\
0 \\
0 \\
-16 \\
0 \\
0 \\
0 \\
0 \\
0 \\
0 \\
0 \\
0\end{array}$ & $\begin{array}{c}0 \\
-16 \\
0 \\
0 \\
0 \\
0 \\
0 \\
16 \\
0 \\
16 \\
0 \\
0 \\
0 \\
0 \\
0 \\
-16 \\
0 \\
0 \\
0 \\
-8 \\
8 \\
8 \\
8 \\
-16 \\
-8 \\
-24 \\
8 \\
8 \\
0 \\
0 \\
16 \\
0 \\
-8 \\
0 \\
16 \\
0 \\
-16 \\
0 \\
8 \\
0 \\
8 \\
-8 \\
0 \\
-8 \\
16 \\
-8 \\
0 \\
24 \\
0 \\
-8 \\
-16\end{array}$ & $\begin{array}{c}0 \\
0 \\
-16 \\
-16 \\
0 \\
0 \\
-16 \\
16 \\
0 \\
0 \\
16 \\
-16 \\
0 \\
0 \\
16 \\
16 \\
0 \\
-16 \\
-8 \\
8 \\
16 \\
0 \\
-8 \\
8 \\
-8 \\
-16 \\
0 \\
8 \\
-8 \\
0 \\
8 \\
0 \\
-8 \\
16 \\
8 \\
0 \\
8 \\
-16 \\
-8 \\
16 \\
8 \\
8 \\
0 \\
0 \\
-8 \\
16 \\
0 \\
-16 \\
0 \\
0 \\
-8\end{array}$ & $\begin{array}{c}0 \\
0 \\
0 \\
16 \\
0 \\
-16 \\
0 \\
0 \\
0 \\
0 \\
0 \\
-16 \\
0 \\
16 \\
0 \\
0 \\
0 \\
0 \\
-16 \\
-8 \\
8 \\
-8 \\
-8 \\
16 \\
8 \\
-8 \\
-24 \\
8 \\
16 \\
0 \\
-16 \\
0 \\
8 \\
0 \\
0 \\
0 \\
0 \\
0 \\
-8 \\
0 \\
8 \\
-8 \\
0 \\
24 \\
16 \\
-8 \\
0 \\
8 \\
0 \\
8 \\
-16\end{array}$ & $\begin{array}{c}0 \\
8 \\
-8 \\
8 \\
-16 \\
8 \\
-8 \\
8 \\
0 \\
8 \\
-8 \\
8 \\
-16 \\
8 \\
-8 \\
8 \\
-8 \\
-8 \\
-8 \\
0 \\
0 \\
0 \\
0 \\
8 \\
0 \\
0 \\
0 \\
0 \\
-8 \\
8 \\
8 \\
8 \\
0 \\
-8 \\
-8 \\
8 \\
-8 \\
-8 \\
0 \\
-8 \\
0 \\
0 \\
-8 \\
0 \\
8 \\
0 \\
16 \\
0 \\
8 \\
0 \\
8\end{array}$ & $\begin{array}{c}0 \\
16 \\
0 \\
-16 \\
0 \\
0 \\
0 \\
0 \\
-16 \\
0 \\
16 \\
0 \\
16 \\
0 \\
-16 \\
0\end{array}$ & $\begin{array}{r}0 \\
8 \\
0 \\
-8 \\
0 \\
-8 \\
0 \\
8 \\
0 \\
8 \\
0 \\
-8 \\
0 \\
-8 \\
0 \\
8 \\
-8 \\
-8 \\
-8 \\
-8 \\
-8 \\
8 \\
8 \\
8 \\
8 \\
8 \\
-8 \\
-8 \\
-8 \\
0 \\
8 \\
0 \\
8 \\
0 \\
8 \\
-32 \\
8 \\
0 \\
8 \\
8 \\
-8 \\
-8 \\
8 \\
-8 \\
-8 \\
-8 \\
0 \\
8 \\
32 \\
\end{array}$ & $\begin{array}{c}16 \\
16 \\
16 \\
0 \\
0 \\
0 \\
0 \\
0 \\
16 \\
0 \\
-16 \\
0 \\
0 \\
0 \\
16 \\
-16 \\
0 \\
-16 \\
0 \\
16 \\
0 \\
-16 \\
0 \\
16 \\
0\end{array}$ \\
\hline
\end{tabular}

2. Applications. It is known that $c_{0} \equiv-1(\bmod 8)$ and that $c_{2} \equiv m(\bmod 4)$; see [8, p. 338], [4, Theorems 3.5 and 3.6]. With the use of the tables in Section 3, the values of $c_{0}$ and $c_{2}$ have been characterized [7] modulo 16 and 8 , respectively. For example, in the case that $2 \mid f$ and $8 \mid m$ (so that $4 \mid b$ by [1] or [3, Theorem 3.15]), we have

$$
\begin{array}{lll}
c_{0} \equiv-1(\bmod 16) & \text { and } \quad c_{2} \equiv 0(\bmod 8), & \text { if } 8 \mid b, \\
c_{0} \equiv 7(\bmod 16) & \text { and } \quad c_{2} \equiv 4(\bmod 8), & \text { otherwise. }
\end{array}
$$

The tables in Section 3 have also been applied [7] to give an elementary proof of the important relation

$$
y \equiv 2 b+m \quad(\bmod 16) .
$$

Hasse [9, p. 232] deduced (1) using deep methods from class field theory. Whiteman [17, Section 5] gave an elementary proof of (1) in the case $81 \mathrm{~m}$ and, thereby, supplied a relatively simple demonstration of the Cunningham-Aigner criterion for the sixteenth power residue character of 2 . In the case $8 \nmid m$, (1) leads to reso- 
TABLE $2 \mathrm{a} . \quad f$ even, $m \equiv \pm 2(\bmod 8)$

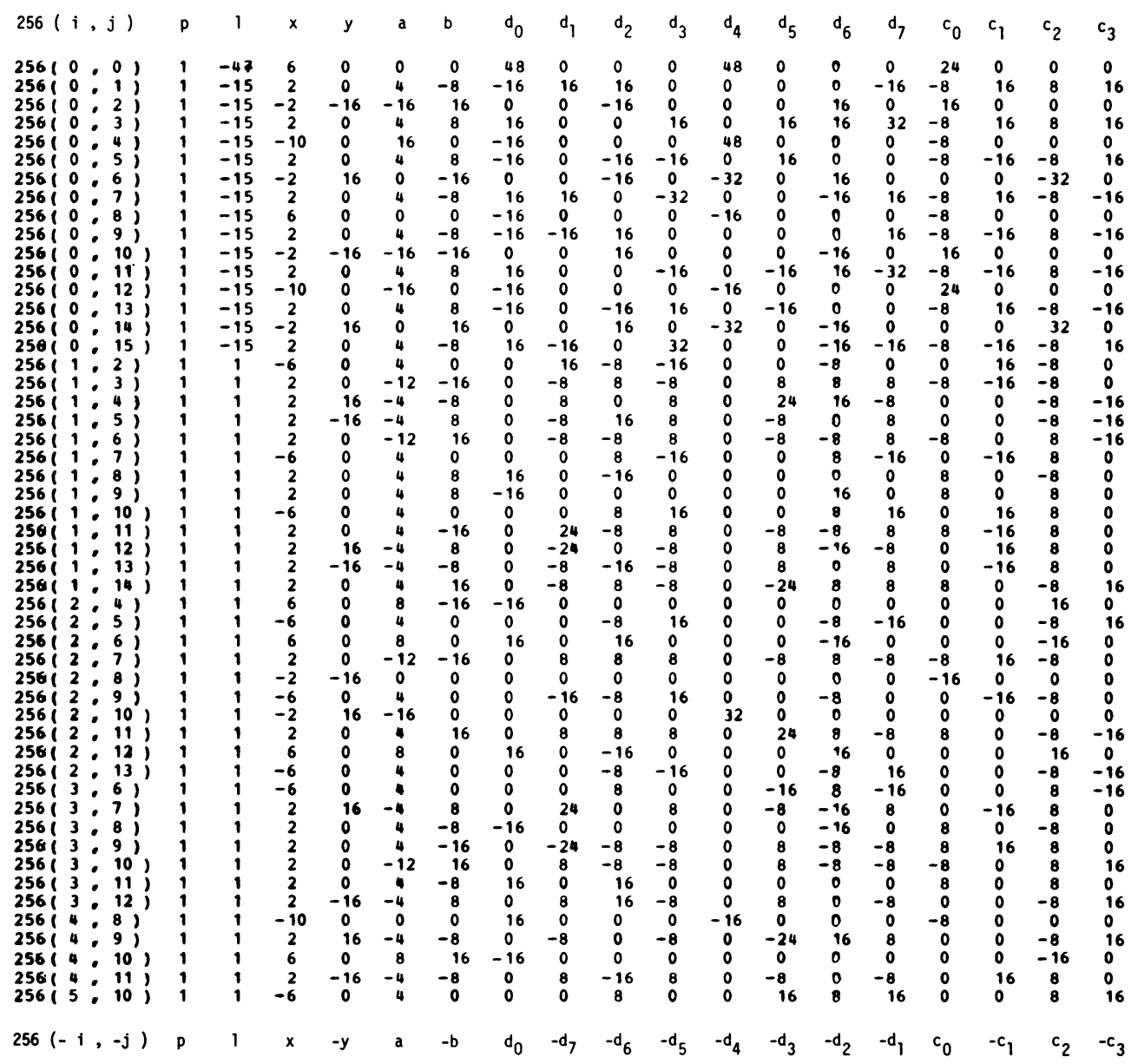

lutions of sign ambiguities in quartic and octic Jacobi and Jacobsthal sums. For example, in the case that 2 is a quartic but not octic residue $(\bmod p)$, i.e., $m \equiv \pm 4$ ( $\bmod 16)$, the sign of $y$ is determined by (1), because in this case $4 \mid b$ by [1] or [3, Theorem 3.15]. This sign determination extends the results of E. Lehmer for quartic sums [11], [12]. Sign ambiguities in octic sums have been resolved only in the case that 2 is a quartic nonresidue $(\bmod p)$, i.e., $m \equiv \pm 2(\bmod 8)$. In this case, the sign of $b$ is determined by $(1)$, since here $y \equiv-m(\bmod 8)$ by $[11$, p. 108] .

Consider now primes $p \equiv 1(\bmod 32)$. For such primes, Hasse $[9$, p. 233] proved that

$$
y+2 b-4\left(c_{1}+c_{3}\right) \equiv m(\bmod 32)
$$

In the case that $y \equiv m \equiv 0(\bmod 16)$, Hasse showed that $(2)$ yields a simple unam- 
TABLE $2 \mathrm{~b} . \quad f$ odd,$m \equiv \pm 2(\bmod 8)$

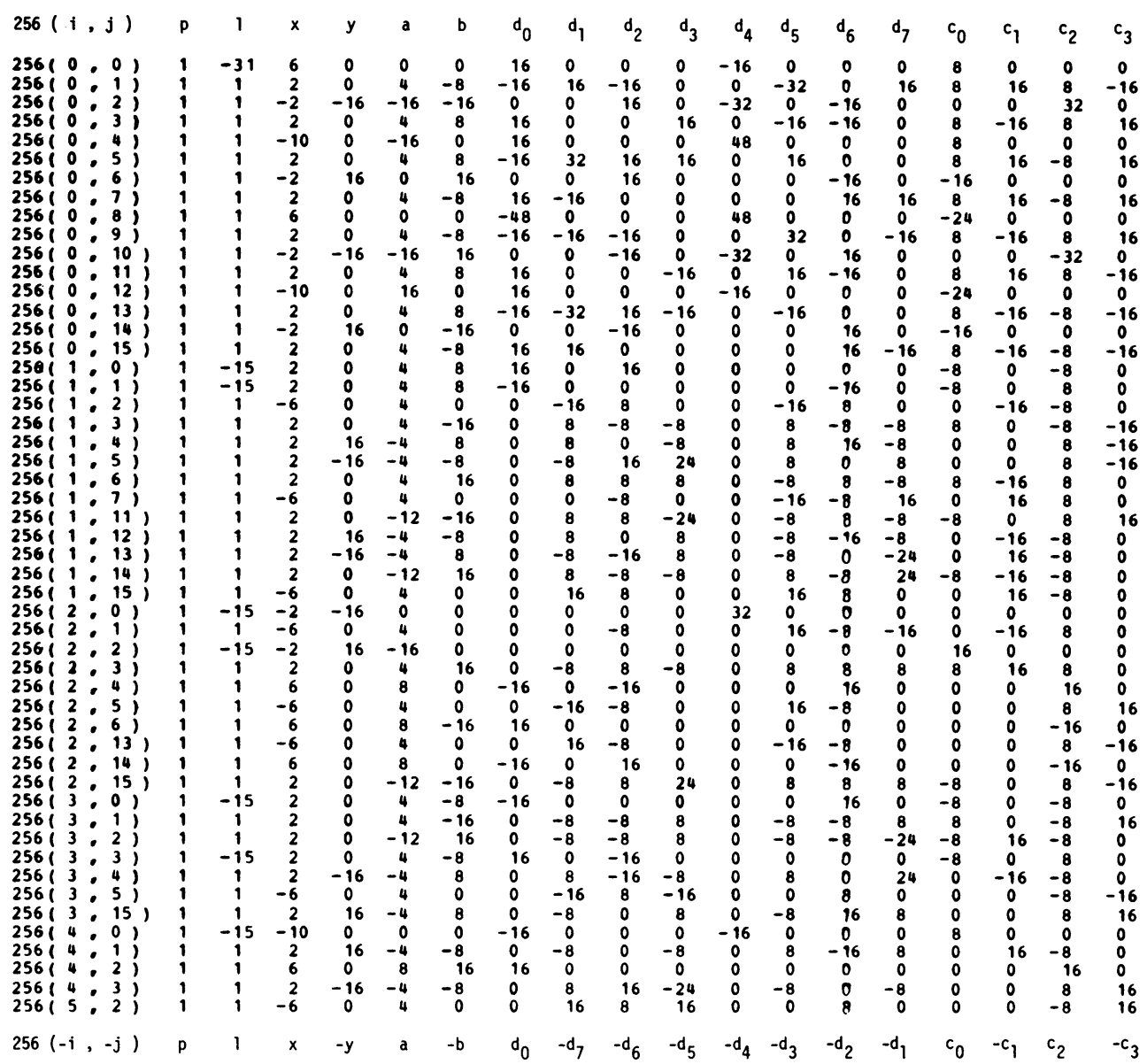

biguous criterion for the 32nd power residue character of 2 . In the case that $4+\mathrm{m}$, (2) leads [7] to a resolution of signs for bioctic Jacobi and Jacobsthal sums. (The latter sums are evaluated up to sign in [4, Theorem 3.9].) No elementary proof of (2) appears to be known, but such a proof could undoubtedly be found once a table of cyclotomic numbers or order 32 has been constructed. Note that (1) and (2) are special cases of J. B. Muskat's equation (9) on p. 146 of Computers in Number Theory, Academic Press, New York, 1971.

3. Tables of $(i, j)$. Of the 256 possible formulas for $(i, j)$ corresponding to a given prime $p$, at most 51 are distinct. These formulas are given in Tables 1a-3a for $f$ even, and in Tables $1 \mathrm{~b}-3 \mathrm{~b}$ for $f$ odd. Tables $1 \mathrm{a}, 1 \mathrm{~b}$ (resp., 3a, 3b) treat the primes $p$ for which $m \equiv 0(\bmod 8)($ resp., $m \equiv 4(\bmod 8))$. Tables $2 \mathrm{a}, 2 \mathrm{~b}$ treat the 
TABLE 3a. $f$ even, $m \equiv 4(\bmod 8)$

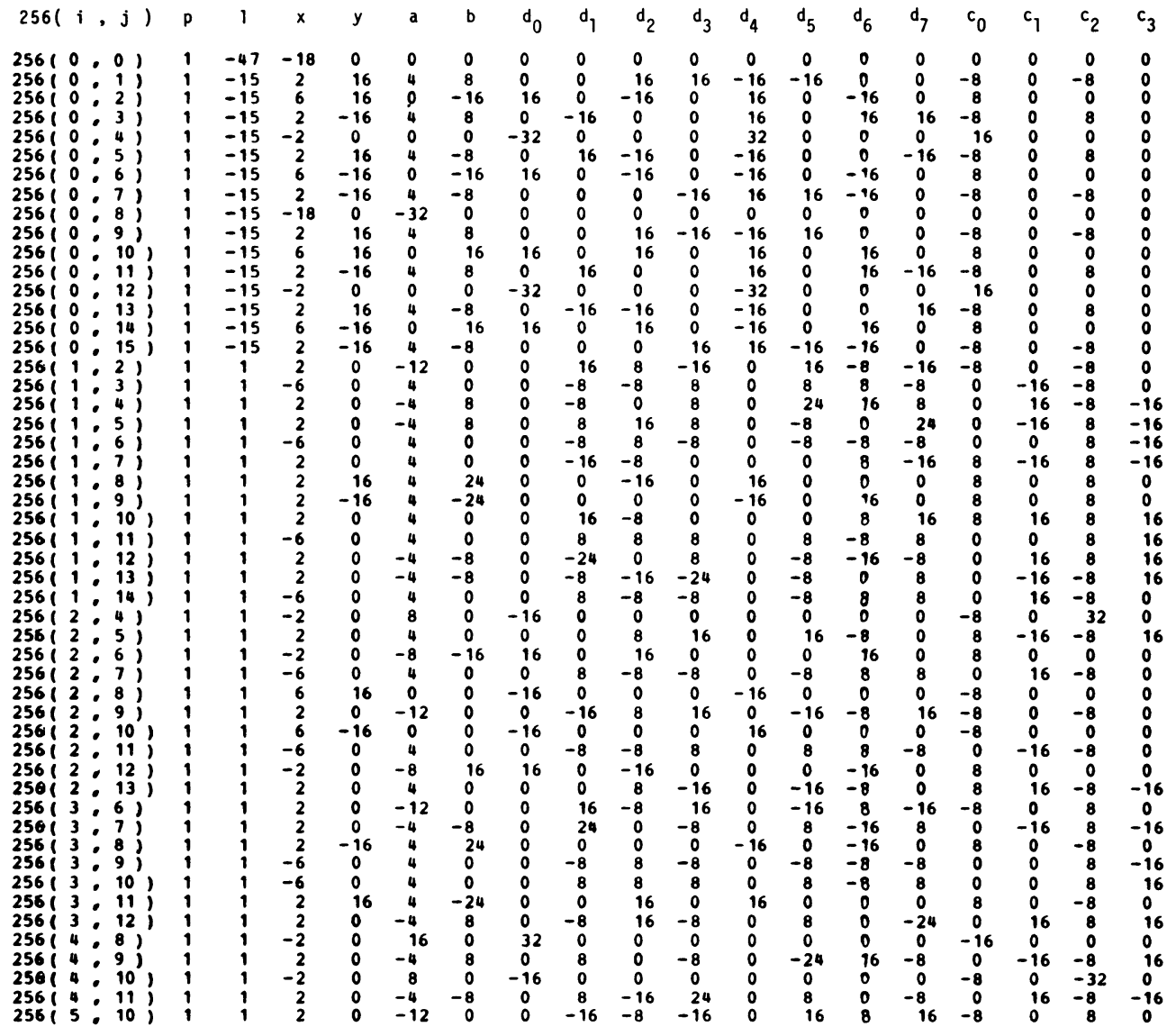

cases $m \equiv \pm 2(\bmod 8)$, where the upper headings are used for $m \equiv 2(\bmod 8)$ and the lower headings are used for $m \equiv-2(\bmod 8)$. For example, from Table $2 a$, we find that

$$
256(0,2)=p-15-2 x-16 y-16 a+16 b-16 d_{2}+16 d_{6}+16 c_{0} \text {, }
$$

when $m \equiv 2(\bmod 8)$, whereas

$$
\begin{aligned}
256(0,2) & =256(0,-14) \\
& =p-15-2 x-16 y-16 b+16 d_{2}-16 d_{6}+32 d_{4}+32 c_{2},
\end{aligned}
$$

when $m \equiv-2(\bmod 8)$. To obtain a formula for a number $(i, j)$ not listed in a given one of Tables $1 a-3 a$ or $1 b-3 b$, one would consult Table $4 a$ or $4 b$ according as $f$ is even or odd. For example, from Table $4 b$, one finds that $(11,4)=(1,13)$. 
TABLE 3 b. $f$ odd, $m \equiv 4(\bmod 8)$

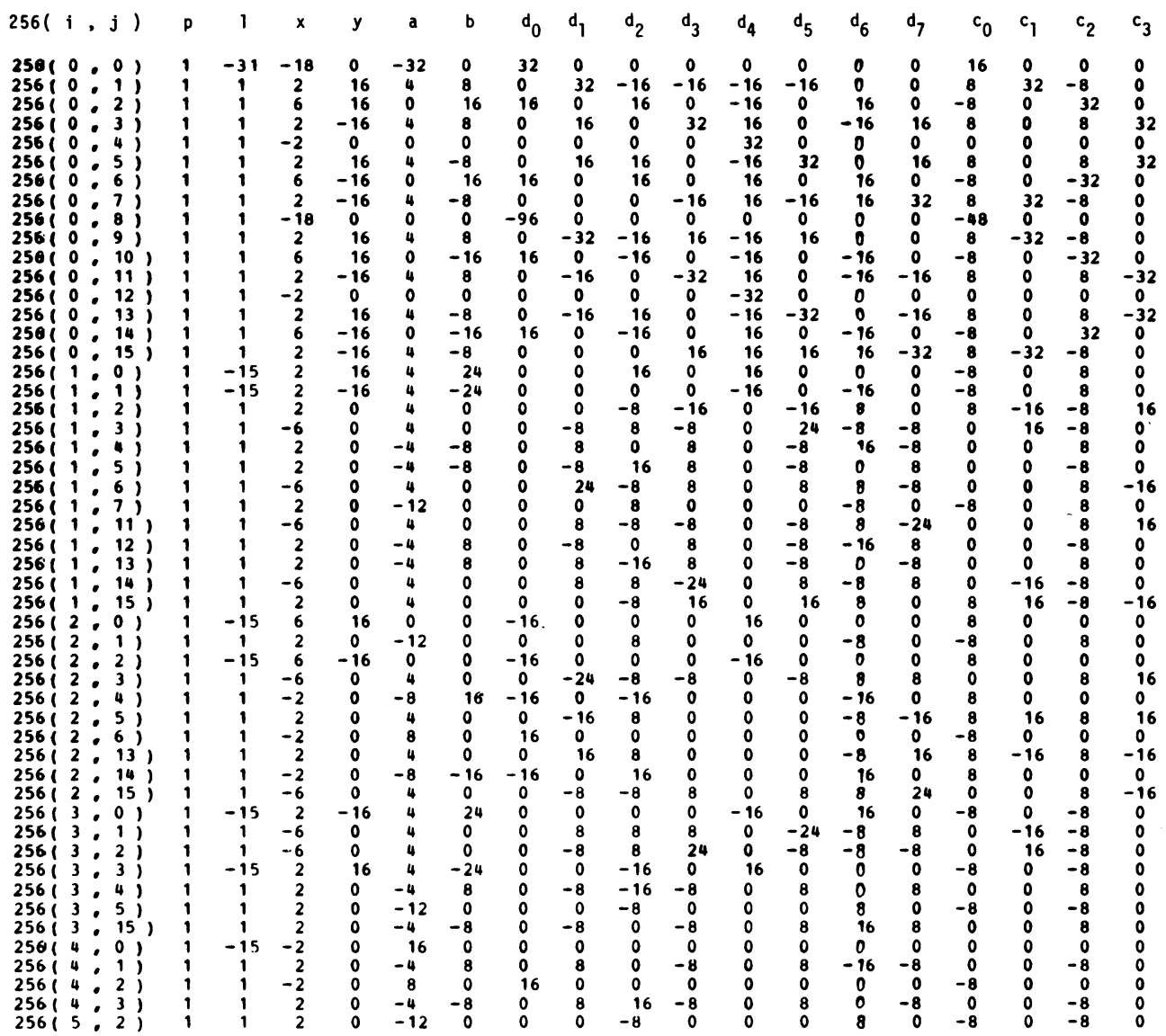

TABLE 4a. $f$ even

$\begin{array}{lllllllllllllllll}\text { i }^{j} & 0 & 1 & 2 & 3 & 4 & 5 & 6 & 7 & 8 & 9 & 10 & 11 & 12 & 13 & 14 & 15 \\ 0 & 0,0 & 0,1 & 0,2 & 0,3 & 0,4 & 0,5 & 0,6 & 0,7 & 0,8 & 0,9 & 0,10 & 0,11 & 0,12 & 0,13 & 0,14 & 0,15 \\ 1 & 0,1 & 0,15 & 1,2 & 1,3 & 1,4 & 1,5 & 1,6 & 1,7 & 1,8 & 1,9 & 1,10 & 1,11 & 1,12 & 1,13 & 1,14 & 1,2 \\ 2 & 0,2 & 1,2 & 0,14 & 1,14 & 2,4 & 2,5 & 2,6 & 2,7 & 2,8 & 2,9 & 2,10 & 2,11 & 2,12 & 2,13 & 2,4 & 1,3 \\ 3 & 0,3 & 1,3 & 1,14 & 0,13 & 1,13 & 2,13 & 3,6 & 3,7 & 3,8 & 3,9 & 3,10 & 3,11 & 3,12 & 3,6 & 2,5 & 1,4 \\ 4 & 0,4 & 1,4 & 2,4 & 1,13 & 0,12 & 1,12 & 2,12 & 3,12 & 4,8 & 4,9 & 4,10 & 4,11 & 4,8 & 3,7 & 2,6 & 1,5 \\ 5 & 0,5 & 1,5 & 2,5 & 2,13 & 1,12 & 0,11 & 1,11 & 2,11 & 3,11 & 4,11 & 5,10 & 5,10 & 4,9 & 3,8 & 2,7 & 1,6 \\ 6 & 0,6 & 1,6 & 2,6 & 3,6 & 2,12 & 1,11 & 0,10 & 1,10 & 2,10 & 3,10 & 4,10 & 5,10 & 4,10 & 3,9 & 2,8 & 1,7 \\ 7 & 0,7 & 1,7 & 2,7 & 3,7 & 3,12 & 2,11 & 1,10 & 0,9 & 1,9 & 2,9 & 3,9 & 4,9 & 4,11 & 3,10 & 2,9 & 1,8 \\ 8 & 0,8 & 1,8 & 2,8 & 3,8 & 4,8 & 3,11 & 2,10 & 1,9 & 0,8 & 1,8 & 2,8 & 3,8 & 4,8 & 3,11 & 2,10 & 1,9 \\ 9 & 0,9 & 1,9 & 2,9 & 3,9 & 4,9 & 4,11 & 3,10 & 2,9 & 1,8 & 0,7 & 1,7 & 2,7 & 3,7 & 3,12 & 2,11 & 1,10 \\ 10 & 0,10 & 1,10 & 2,10 & 3,10 & 4,10 & 5,10 & 4,10 & 3,9 & 2,8 & 1,7 & 0,6 & 1,6 & 2,6 & 3,6 & 2,12 & 1,11 \\ 11 & 0,11 & 1,11 & 2,11 & 3,11 & 4,11 & 5,10 & 5,10 & 4,9 & 3,8 & 2,7 & 1,6 & 0,5 & 1,5 & 2,5 & 2,13 & 1,12 \\ 12 & 0,12 & 1,12 & 2,12 & 3,12 & 4,8 & 4,9 & 4,10 & 4,11 & 4,8 & 3,7 & 2,6 & 1,5 & 0,4 & 1,4 & 2,4 & 1,13 \\ 13 & 0,13 & 1,13 & 2,13 & 3,6 & 3,7 & 3,8 & 3,9 & 3,10 & 3,11 & 3,12 & 3,6 & 2,5 & 1,4 & 0,3 & 1,3 & 1,14 \\ 14 & 0,14 & 1,14 & 2,4 & 2,5 & 2,6 & 2,7 & 2,8 & 2,9 & 2,10 & 2,11 & 2,12 & 2,13 & 2,4 & 1,3 & 0,2 & 1,2 \\ 15 & 0,15 & 1,2 & 1,3 & 1,4 & 1,5 & 1,6 & 1,7 & 1,8 & 1,9 & 1,10 & 1,11 & 1,12 & 1,13 & 1,14 & 1,2 & 0,1\end{array}$


TABLE 4b. $f$ odd

$\begin{array}{lrrrrrrrrrrrrrrrr}\text { i j } & 0 & 1 & 2 & 3 & 4 & 5 & 6 & 7 & 8 & 9 & 10 & 11 & 12 & 13 & 14 & 15 \\ 0 & 0,0 & 0,1 & 0,2 & 0,3 & 0,4 & 0,5 & 0,6 & 0,7 & 0,8 & 0,9 & 0,10 & 0,11 & 0,12 & 0,13 & 0,14 & 0,15 \\ 1 & 1,0 & 1,1 & 1,2 & 1,3 & 1,4 & 1,5 & 1,6 & 1,7 & 0,9 & 0,7 & 1,7 & 1,11 & 1,12 & 1,13 & 1,14 & 1,15 \\ 2 & 2,0 & 2,1 & 2,2 & 2,3 & 2,4 & 2,5 & 2,6 & 1,11 & 0,10 & 1,7 & 0,6 & 1,6 & 2,6 & 2,13 & 2,14 & 2,15 \\ 3 & 3,0 & 3,1 & 3,2 & 3,3 & 3,4 & 3,5 & 2,13 & 1,12 & 0,11 & 1,11 & 1,6 & 0,5 & 1,5 & 2,5 & 3,5 & 3,15 \\ 4 & 4,0 & 4,1 & 4,2 & 4,3 & 4,0 & 3,15 & 2,14 & 1,13 & 0,12 & 1,12 & 2,6 & 1,5 & 0,4 & 1,4 & 2,4 & 3,4 \\ 5 & 3,3 & 4,3 & 5,2 & 5,2 & 4,1 & 3,0 & 2,15 & 1,14 & 0,13 & 1,13 & 2,13 & 2,5 & 1,4 & 0,3 & 1,3 & 2,3 \\ 6 & 2,2 & 3,2 & 4,2 & 5,2 & 4,2 & 3,1 & 2,0 & 1,15 & 0,14 & 1,14 & 2,14 & 3,5 & 2,4 & 1,3 & 0,2 & 1,2 \\ 7 & 1,1 & 2,1 & 3,1 & 4,1 & 4,3 & 3,2 & 2,1 & 1,0 & 0,15 & 1,15 & 2,15 & 3,15 & 3,4 & 2,3 & 1,2 & 0,1 \\ 8 & 0,0 & 1,0 & 2,0 & 3,0 & 4,0 & 3,3 & 2,2 & 1,1 & 0,0 & 1,0 & 2,0 & 3,0 & 4,0 & 3,3 & 2,2 & 1,1 \\ 9 & 1,0 & 0,15 & 1,15 & 2,15 & 3,15 & 3,4 & 2,3 & 1,2 & 0,1 & 1,1 & 2,1 & 3,1 & 4,1 & 4,3 & 3,2 & 2,1 \\ 10 & 2,0 & 1,15 & 0,14 & 1,14 & 2,14 & 3,5 & 2,4 & 1,3 & 0,2 & 1,2 & 2,2 & 3,2 & 4,2 & 5,2 & 4,2 & 3,1 \\ 11 & 3,0 & 2,15 & 1,14 & 0,13 & 1,13 & 2,13 & 2,5 & 1,4 & 0,3 & 1,3 & 2,3 & 3,3 & 4,3 & 5,2 & 5,2 & 4,1 \\ 12 & 4,0 & 3,15 & 2,14 & 1,13 & 0,12 & 1,12 & 2,6 & 1,5 & 0,4 & 1,4 & 2,4 & 3,4 & 4,0 & 4,1 & 4,2 & 4,3 \\ 13 & 3,3 & 3,4 & 3,5 & 2,13 & 1,12 & 0,11 & 1,11 & 1,6 & 0,5 & 1,5 & 2,5 & 3,5 & 3,15 & 3,0 & 3,1 & 3,2 \\ 14 & 2,2 & 2,3 & 2,4 & 2,5 & 2,6 & 1,11 & 0,10 & 1,7 & 0,6 & 1,6 & 2,6 & 2,13 & 2,14 & 2,15 & 2,0 & 2,1 \\ 15 & 1,1 & 1,2 & 1,3 & 1,4 & 1,5 & 1,6 & 1,7 & 0,9 & 0,7 & 1,7 & 1,11 & 1,12 & 1,13 & 1,14 & 1,15 & 1,0\end{array}$

Department of Mathematics

University of California, San Diego

La Jolla, California 92093

Department of Applied Physics and Information Sciences University of California, San Diego

La Jolla, California 92093

1. P. BARRUCAND \& H. COHN, "Note on primes of the form $x^{2}+32 y^{2}$, class number, and residuacity," J. Reine Angew. Math., v. 238, 1969 , pp. 67-70.

2. L. BAUMERT \& H. FREDERICKSEN, "The cyclotomic numbers of order eighteen with applications to difference sets," Math. Comp., v. 21, 1967, pp. 204-219.

3. B. C. BERNDT \& R. J. EVANS, "Sums of Gauss, Jacobi, and Jacobsthal," J. Number Theory. (To appear.)

4. B. C. BERNDT \& R. J. EVANS, "Sums of Gauss, Eisenstein, Jacobi, Jacobsthal, and Brewer," Illinois J. Math. (To appear.)

5. L. E. DICKSON, "Cyclotomy, higher congruences, and Waring's problem," Amer. J. Math., v. 57, 1935 , pp. $391-424$.

6. R. J. EVANS, "Bioctic Gauss sums and sixteenth power residue difference sets," Acta Arith. (To appear.)

7. R. J. EVANS, "Resolution of sign ambiguities of Jacobi and Jacobsthal sums," Pacific J. Math. (To appear.)

8. R. GIUDICI, J. MUSKAT \& S. ROBINSON, “On the evaluation of Brewer's character sums," Trans. Amer. Math. Soc., v. 171, 1972, pp. 317-347.

9. H. HASSE, "Der $2^{n}$-te Potenzcharakter von 2 im Körper der $2^{n}$-ten Einheitswurzeln," Rend. Circ. Mat. Palermo Ser. II, v. 7, 1958, pp. 185-243.

10. E. LEHMER, "On the cyclotomic numbers of order sixteen," Canad. J. Math., v. 6, 1954 , pp. $449-454$.

11. E. LEHMER, “On the number of solutions of $u^{k}+D \equiv w^{2}(\bmod p)$," Pacific J. Math., v. 5, 1955, pp. 103-118.

12. E. LEHMER, “On Jacobi functions,” Pacific J. Math., v. 10, 1960, pp. 887-893.

13. P. A. LEONARD \& K. S. WILLIAMS, "The cyclotomic numbers of order eleven," Acta Arith., v. 26, 1975 , pp. 367-383.

14. P. A. LEONARD \& K. S. WILLIAMS, "The cyclotomic numbers of order seven," Proc. Amer. Math. Soc., v. 51, 1975, pp. 295-300. 
15. J. B. MUSKAT, "The cyclotomic numbers of order fourteen," Acta Arith., v. 11, 1966, pp. 263-279.

16. J. B. MUSKAT \& A. L. WHITEMAN, "The cyclotomic numbers of order twenty," Acta Arith., v. 17, 1970, pp. 185-216.

17. A. L. WHITEMAN, "The cyclotomic numbers of order sixteen," Trans Amer. Math. Soc., v. 86, 1957, pp. 401-413.

18. A. L. WHITEMAN, "The cyclotomic numbers of order ten," Proc. Sympos. Appl. Math., vol. 10, Amer. Math. Soc., Providence, R. I., 1960, pp. 95-111.

19. A. L. WHITEMAN, "The cyclotomic numbers of order twelve," Acta Artth., v. 6, 1960, pp. 53-76. 